\title{
A comparison of in-vitro fertilization of rabbit eggs using spermatozoa recovered from the uterus or vagina*
}

\author{
Pramila Dandekar and Lynn Fraser \\ Department of Obstetrics and Gynecology, Yale University School of Medicine, \\ New Haven, Connecticut 06510, U.S.A.
}

\begin{abstract}
Summary. Spermatozoa were recovered from the uterine horns or vagina of mated rabbit does 12-20 hr p.c. and their fertilizing capacity tested in an in-vitro system. Uterine spermatozoa gave consistently high fertilization $(93-100 \%)$ throughout. Vaginal spermatozoa gave good results $(81-85 \%) 12-14 \mathrm{hr}$ p.c. but fertilization then declined with time. Vaginal spermatozoa recovered from mated females with or without ligation of the uterine horns were equally able to fertilize eggs. Fragmentation of uncleaved eggs was noted in experiments with vaginal spermatozoa, but never with uterine spermatozoa. The former were slower in penetrating eggs and the zygotes were correspondingly slower in cleaving. Triploidy, possibly due to ageing, was found in approximately $15 \%$ of the embryos fertilized by vaginal spermatozoa.
\end{abstract}

\section{Introduction}

The ability of the vagina to capacitate spermatozoa was first reported by Dauzier \& Thibault (1956) who obtained fertilization of rabbit eggs in-vitro with spermatozoa recovered from the vagina 12 hr after mating. Results obtained after tubal insemination with spermatozoa recovered from the vaginae of non-ligated and cervically ligated female rabbits mated 12-15 hr earlier led Bedford (1967) to conclude that rabbit spermatozoa may undergo a considerable degree of capacitation in the vagina as a result of secretions descending from the uterus. Using an in-vitro fertilization system, Chang et al. (1971) reported that a higher rate of fertilization was achieved with vaginal than uterine spermatozoa. They postulated that this might be due to a higher concentration of spermatozoa in the vaginal flushings.

A very high rate of fertilization in-vitro $(90-100 \%)$ has been consistently achieved with uterine spermatozoa in our laboratory (Fraser et al., 1971). In this study, experiments were carried out to determine the fertilizing ability of vaginal spermatozoa recovered at various times after mating. In addition, the influence of uterine fluid on vaginal spermatozoa and the chromosomal state of the resulting embryos were examined.

\section{Materials and Methods}

The defined medium of Brackett \& Williams (1968) was used for recovery and fertilization of gametes. All manipulations were carried out at $37^{\circ} \mathrm{C}$. Egg donors were New Zealand white rabbits, individually caged for at least 3 weeks. They were induced to superovulate with $1 \mathrm{mg} \mathrm{FSH} \mathrm{(Armour)/day} \mathrm{for} 3$ days followed by $2.5 \mathrm{mg} \mathrm{LH}$ (Armour) $13 \mathrm{hr}$ before death (Fraser et al., 1971). Unfertilized tubal eggs were recovered and follicle cells were removed as described by Fraser et al. (1971). Spermatozoa were recovered from females mated with three fertile males and killed at 2-hr intervals $10-20 \mathrm{hr}$ post coitum (p.c.). For recovery of uterine and vaginal spermatozoa, the cervical-vaginal junction was clamped; the uterine horns were flushed with $4 \mathrm{ml}$ medium and the vagina with $2 \mathrm{ml}$. To rule out the possibility that the uterine secretions present in the vagina influence capacitation, in some oestrous females the uterine horns were bilaterally ligated just proximal to the cervix. After 8-15

* Reprint requests to Dr L. Fraser, Clinical Research Centre, Watford Road, Harrow, Middlesex HA1 3UJ, England. 
days, the females with a ligated cervix were each mated with three fertile males and killed $12-14 \mathrm{hr}$ p.c. At the time of collection of vaginal spermatozoa, the effectiveness of the cervical ligation was judged by the presence of fluid within the uterine horns $(2-8 \mathrm{ml} / \mathrm{horn})$. Uterine fluid was examined for the presence of spermatozoa and, as a further check, the oviducts of the females were flushed and the eggs checked for fertilization.

Sperm counts were within the range of $30,000-380,000 / \mathrm{ml}$ for uterine and vaginal sperm samples. Motility was generally $60-90 \%$. With older sperm samples, the values tended to be at lower end of the range, but they were within the range expected to give a high rate of fertilization in vitro (unpublished results).

Uterine and vaginal sperm flushings were placed in separate culture dishes and denuded eggs were added. After incubation for $4 \mathrm{hr}$, eggs were transferred to culture medium (Brackett \& Williams, 1968). Subsequent culture and chromosome preparation techniques have been fully described elsewhere (Fraser et al., 1971).

Because initial observations suggested that eggs mixed with vaginal spermatozoa were slow to cleave, some eggs were transferred to culture medium at specific times (2,3 or $4 \mathrm{hr})$ after mixing of gametes. This was done with vaginal and uterine sperm suspensions to allow comparison of timing of sperm penetration and subsequent cleavage. The eggs were observed after 5 and $6 \mathrm{hr}$ for pronuclear formation and again after $22-26 \mathrm{hr}$ for cleavage. Cleaved embryos were processed for visualization of chromosomes.

\section{Results}

Fertilization rates obtained with uterine spermatozoa recovered $12-20 \mathrm{hr}$ p.c. were consistently high, with no significant differences $\left(\chi^{2}, P>0.05\right)$ between the 2-hr intervals (Table 1). Vaginal spermatozoa recovered from the same females at the same intervals gave lower rates. The highest fertilization rate using vaginal spermatozoa was obtained $12-14 \mathrm{hr}$ p.c.; although the proportion of eggs cleaving $(81.3 \%)$ was lower than for uterine spermatozoa recovered at the same time $(92.8 \%)$, the difference was not significant. As the age of the vaginal spermatozoa increased, the fertilization rate decreased and was significantly lower than that obtained with uterine spermatozoa (Table 1).

In addition to the reduced fertilization rate with vaginal spermatozoa, a proportion of the eggs (approximately $10-15 \%$ with $12-18 \mathrm{hr}$ spermatozoa and over $50 \%$ with $18-20 \mathrm{hr}$ spermatozoa) failed to cleave and had fragmented when examined $24-26 \mathrm{hr}$ after mixing. None of the eggs mixed with the uterine spermatozoa fragmented (Table 1).

Uterine ligation made no significant difference in the fertilizing ability of vaginal spermatozoa recovered at 12-14 hr p.c. (Table 2). Fragmentation was again noted in 10-15\% of eggs. The

Table 1. Fertilization in vitro of rabbit eggs with uterine and vaginal spermatozoa recovered at various times after mating. The results for each time interval represent 3 experiments and the sperm counts for individual experiments are given

\begin{tabular}{|c|c|c|c|c|}
\hline & \multicolumn{4}{|c|}{ Time of sperm recovery (hr p.c.) } \\
\hline & $12-14$ & $14-16$ & $16-18$ & $18-20$ \\
\hline \multicolumn{5}{|l|}{ Uterine spermatozoa } \\
\hline Sperm count $\left(\times 10^{5}\right)$ & $2 \cdot 3,1 \cdot 5,1 \cdot 7$ & $1 \cdot 8,1 \cdot 2,1 \cdot 0$ & $0 \cdot 7,0 \cdot 8,1 \cdot 2$ & $1 \cdot 1,1 \cdot 1,0.9$ \\
\hline Total no. of eggs & 14 & 16 & 23 & 23 \\
\hline No. cleaved & $13(92 \cdot 8 \%)$ & $16(100 \%)$ & $23(100 \%)$ & $22(95 \cdot 6 \%)$ \\
\hline No. fragmented & 0 & 0 & 0 & 0 \\
\hline \multicolumn{5}{|l|}{ Vaginal spermatozoa } \\
\hline Sperm count $\left(\times 10^{5}\right)$ & $2 \cdot 0,2 \cdot 8,2 \cdot 4$ & $3 \cdot 8,1 \cdot 2,1 \cdot 8$ & $0 \cdot 3,1 \cdot 0,1 \cdot 0$ & $1 \cdot 2,0 \cdot 8,1 \cdot 1$ \\
\hline Total no. of eggs & 32 & 53 & 67 & 55 \\
\hline No. cleaved & $26(81 \cdot 3 \%)$ & $34(64 \cdot 0 \%)$ & $35(52 \cdot 2 \%)$ & $9(16 \cdot 4 \%)$ \\
\hline No. fragmented & $3(9 \cdot 3 \%)$ & $9(16.9 \%)$ & $8(12 \cdot 0 \%)$ & $32(58 \cdot 2 \%)$ \\
\hline
\end{tabular}


Table 2. Fertilization in vitro of rabbit eggs with uterine and vaginal spermatozoa recovered from intact females, and with vaginal spermatozoa recovered from females with ligated uteri (12-14 hr p.c.)

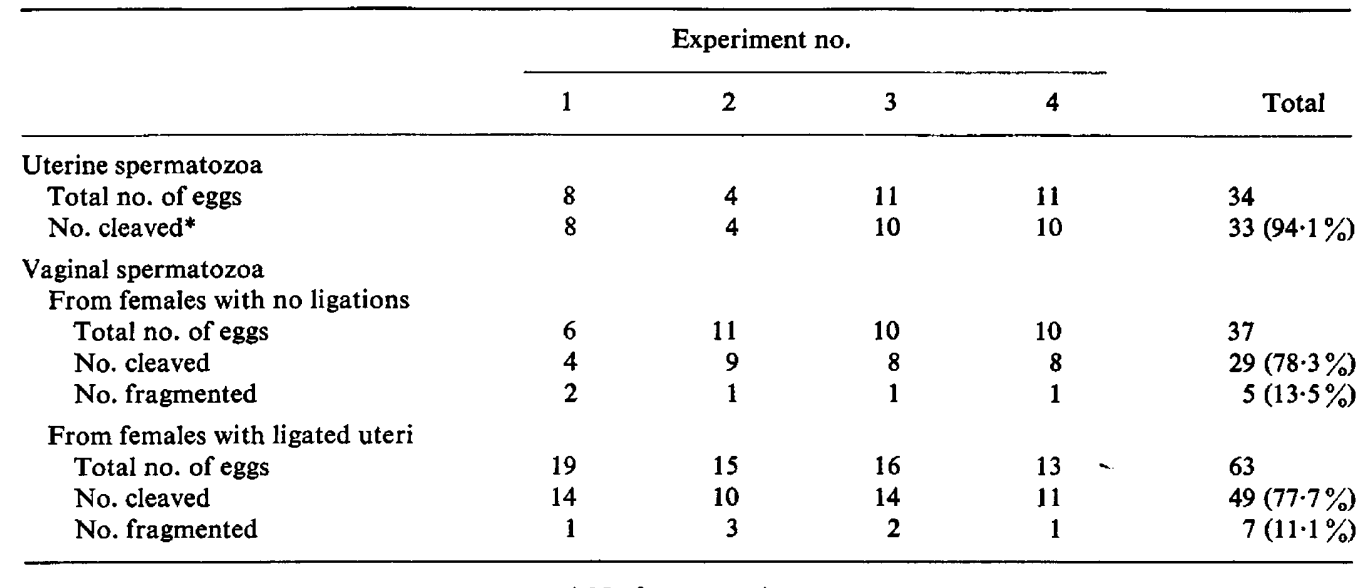

ligations appeared to be effective; in all animals the uterine fluid was free of spermatozoa and none of the eggs was fertilized. One of the non-ligated females was found to be pseudopregnant; no spermatozoa were present in the uterus but the vaginal spermatozoa were recovered and tested. Of the 13 eggs used, 6 cleaved to the two-cell stage and out of 7 uncleaved, 4 fragmented.

When eggs were transferred at intervals from uterine sperm suspensions to culture medium, the majority of eggs had pronuclei 5-6 hr after mixing of gametes. There was no significant difference in the subsequent cleavage rate of eggs transferred after 2,3 or $4 \mathrm{hr}(84 \cdot 2,90$ and $94.2 \%$ respectively; Table 3). In comparison, none of the eggs transferred from vaginal sperm suspensions at the same time intervals showed pronuclei after $5 \mathrm{hr}$ and relatively few after $6 \mathrm{hr}$ (Table 3). There was also a difference in the cleavage rate when eggs were removed from vaginal sperm suspensions after 2,3 or $4 \mathrm{hr}(31.4,58.5$ and $84.6 \%$, respectively). Thus only eggs remaining in the vaginal sperm suspension for the full $4 \mathrm{hr}$ reached the cleavage rate obtained with uterine spermatozoa (Tables 1 and 2).

Examination of metaphase chromosomes of embryos fertilized with uterine spermatozoa recovered at various time intervals indicated that most embryos were diploid (Table 4). In the experiments in which eggs were transferred from uterine sperm suspensions after $2,3 \mathrm{or} 4 \mathrm{hr}$, two of the embryos were triploid ( $4.8 \%$, Table 5). In contrast, embryos cleaved after fertilization with vaginal spermatozoa showed a relatively high level of triploidy (approximately 15\%, Tables 4 and 5).

Table 3. Fertilization in vitro of rabbit eggs transferred from suspensions of uterine and vaginal spermatozoa (12-14 hr p.c.)

\begin{tabular}{|c|c|c|c|c|c|c|}
\hline & \multicolumn{6}{|c|}{ Eggs transferred at: } \\
\hline & \multicolumn{2}{|c|}{$2 \mathrm{hr}$} & \multicolumn{2}{|c|}{$3 \mathrm{hr}$} & \multicolumn{2}{|c|}{$4 \mathrm{hr}$} \\
\hline & Uterine & Vaginal & Uterine & Vaginal & Uterine & Vaginal \\
\hline $\begin{array}{l}\text { Total no. of eggs } \\
\text { No. with pronuclei }\end{array}$ & 38 & 35 & 30 & 41 & 37 & 52 \\
\hline At $5 \mathrm{hr}$ & 25 & $\mathbf{0}$ & 19 & $\mathbf{0}$ & 30 & $\mathbf{0}$ \\
\hline At $6 \mathrm{hr}$ & 27 & 4 & 23 & 10 & 32 & 14 \\
\hline $\begin{array}{l}\text { No. cleaving } \\
\text { At } 22-24 \mathrm{hr} \\
\text { At } 24-26 \mathrm{hr}\end{array}$ & $\begin{array}{c}28+4^{*} \\
32\end{array}$ & $\begin{array}{c}6+5^{*} \\
11\end{array}$ & $\begin{array}{c}25+2^{*} \\
27\end{array}$ & $\begin{array}{c}13+11^{*} \\
24\end{array}$ & $\begin{array}{c}33+2^{*} \\
35\end{array}$ & $\begin{array}{c}29+15^{*} \\
44\end{array}$ \\
\hline Total cleaved (\%) & $84 \cdot 2 \%$ & $31.4 \%$ & $90 \cdot 0 \%$ & $58.5 \%$ & $94.5 \%$ & $84.6 \%$ \\
\hline
\end{tabular}

* Pronuclear. 
Table 4. Chromosome complements of rabbit eggs fertilized in vitro with uterine and vaginal spermatozoa and incubated in the sperm suspension for $4 \mathrm{hr}$ before being transferred to culture medium

\begin{tabular}{|c|c|c|c|c|c|c|c|c|}
\hline \multirow{2}{*}{$\begin{array}{l}\text { Time of sperm } \\
\text { recovery } \\
\text { (hr p.c.) }\end{array}$} & \multicolumn{4}{|c|}{ Uterine spermatozoa } & \multicolumn{4}{|c|}{ Vaginal spermatozoa } \\
\hline & $2 \mathrm{~N}$ & $3 \mathrm{~N}$ & $\begin{array}{c}3 \mathrm{~N} / \text { total } \\
(\%)\end{array}$ & $\begin{array}{l}\text { Lacking } \\
\text { metaphase }\end{array}$ & $2 \mathrm{~N}$ & $3 \mathrm{~N}$ & $\begin{array}{c}3 \mathrm{~N} / \text { total } \\
(\%)\end{array}$ & $\begin{array}{c}\text { Lacking } \\
\text { metaphase }\end{array}$ \\
\hline $12-14$ & 11 & 0 & 0 & 1 & 15 & 3 & $16 \cdot 6$ & 6 \\
\hline $14-16$ & 13 & 0 & 0 & 2 & 18 & 4 & $18 \cdot 1$ & 4 \\
\hline $16-18$ & 12 & 0 & 0 & 1 & 22 & 3 & $12 \cdot 0$ & 5 \\
\hline $18-20$ & 13 & 0 & 0 & 1 & 6 & 0 & $0^{*}$ & 0 \\
\hline
\end{tabular}

* Few eggs fertilized.

Table 5. Chromosome complements of eggs fertilized in vitro with 12-14 hr vaginal and uterine spermatozoa after transfer of eggs to the culture medium $2-4 \mathrm{hr}$ after mixing of gametes

\begin{tabular}{cccccccccc}
\hline $\begin{array}{c}\text { Eggs } \\
\text { transferred } \\
\begin{array}{c}\text { from } \\
\text { egg+sperm }\end{array}\end{array}$ & \multicolumn{4}{c}{ Uterine spermatozoa } & & \multicolumn{3}{c}{ Vaginal spermatozoa } \\
mixture (hr) & $2 \mathrm{~N}$ & $3 \mathrm{~N}$ & $\begin{array}{c}\text { 3N/total } \\
(\%)\end{array}$ & $\begin{array}{c}\text { Lacking } \\
\text { metaphase }\end{array}$ & $2 \mathrm{~N}$ & $3 \mathrm{~N}$ & $\begin{array}{c}\text { 3N/total } \\
(\%)\end{array}$ & $\begin{array}{c}\text { Lacking } \\
\text { metaphase }\end{array}$ \\
\hline 2 & 24 & 0 & 0 & 2 & 8 & 1 & $11 \cdot 1$ & 1 \\
3 & 16 & 1 & $5 \cdot 8$ & 3 & 12 & 3 & $20 \cdot 0$ & 4 \\
4 & 26 & 1 & $3 \cdot 8$ & 4 & 29 & 5 & $14 \cdot 3$ & 5 \\
\hline
\end{tabular}

\section{Discussion}

The fertilization of rabbit eggs in vitro has been successfully achieved with spermatozoa recovered from the uteri of mated does (Chang, 1959; Brackett \& Williams, 1968; Fraser et al., 1971). In the present study, the fertilizing ability of uterine spermatozoa remained consistently high throughout the time period examined (12-20 hr p.c.), while that of vaginal spermatozoa was highest $12-14 \mathrm{hr}$ p.c. and decreased with increasing time of residence in the vagina. These findings are in agreement with other reports that uterine spermatozoa retain their fertilizing ability for a long time (Seitz et al., 1970), while vaginal spermatozoa show optimal fertilizing ability when recovered $12-15 \mathrm{hr}$ p.c. (Dauzier \& Thibault, 1956; Bedford, 1967). The results, however, are not in accord with the in-vitro study of Chang et al. (1971) who reported that vaginal spermatozoa recovered 14-16 hr after mating showed a higher fertilization rate than uterine spermatozoa recovered from the same females. The fertilization rate obtained in their experiments with uterine spermatozoa was quite low (29-33\%) and perhaps the system was not operating under optimal conditions.

The lower cleavage rate obtained with vaginal spermatozoa in the present study perhaps reflects a generally poorer sperm population in the vagina since many spermatozoa, particularly those destined to achieve fertilization in vivo, move up the reproductive tract and do not remain in the vagina (Braden, 1953). The vagina would thus appear to have the least selected population of spermatozoa in the tract. The results also indicate that the vaginal environment is relatively unfavourable for maintaining sperm fertilizing ability for long periods. There may be changes in the vaginal secretions deleterious to eggs or spermatozoa (Morton \& Glover, 1974), although no significant changes in sperm concentration or motility were observed in the vaginal samples obtained from our does killed at different times after mating. The results from the pseudopregnant female suggest that although the pseudopregnant uterus is apparently a hostile environment (spermatozoa have rarely been found in similar females) the vagina still supports a population of motile spermatozoa, some of which are capable of fertilizing eggs. The occurrence of fragmentation in eggs mixed with vaginal spermatozoa, but not uterine spermatozoa was unusual. Although Chang et al. (1971) reported that fragmentation of fertilized and, particularly, unfertilized eggs is very common, this 
has not been the case with the in-vitro system used here. It appears that the fragmentation in the present experiments is associated with vaginal spermatozoa and perhaps reflects a less favourable environment for sperm preservation than the uterus. Enzymes released by damaged spermatozoa could possibly account for the fragmentation.

The results after ligation of the uteri indicate that spermatozoa recovered from the vagina of a non-ligated or ligated female can fertilize equally well, suggesting that capacitation of spermatozoa in the vagina does not require the influence of uterine secretions. This finding is at variance with the observations of Bedford (1967) that vaginal spermatozoa recovered from females with bilaterally ligated uterine horns gave lower fertilization rates than those from females with no ligations. Morton \& Glover (1974) obtained some cleavage of rabbit eggs in vivo when spermatozoa that had been preincubated in an isolated cervix for $8 \mathrm{hr}$ were placed in the oviducts of females injected with HCG $13 \mathrm{hr}$ previously, indicating that the spermatozoa were at least partly, if not totally, capacitated at the time of transfer.

In comparison to uterine spermatozoa, those from the vagina were slower in penetrating eggs and the first cleavage was slightly retarded. This may have been because the spermatozoa were only partly capacitated at the time of recovery, or that some unknown factor present in the vaginal flushings affected sperm penetration. The apparent 3-hr time-lag (Table 3) was perhaps necessary to allow either completion of capacitation or diffusion of some inhibitory vaginal substance. However, attempts to achieve capacitation in vitro of ejaculated rabbit spermatozoa in the same fertilization medium have been completely unsuccessful (unpublished results). Whether partly capacitated sperm could complete capacitation in vitro in this medium is not known.

A low incidence of triploidy ( $4.5 \%$ ) was noted after in-vitro fertilization with uterine spermatozoa (Frazer \& Dandekar, 1973a). The high incidence of triploidy with vaginal spermatozoa (15\%) may have been due to ageing of the eggs in vitro since spermatozoa were slow to penetrate. Similar results were obtained when eggs were aged in vitro for $2 \frac{1}{2} \mathrm{hr}$ and mixed with uterine capacitated spermatozoa (Fraser \& Dandekar, 1973b). No attempts were made to distinguish between dispermy and digyny as the basis for triploidy, but the latter is more often found in rabbits (Austin, 1960).

We thank Ms Kathy Petzold for technical assistance. The work was supported by a Ford Foundation grant.

\section{References}

Austin, C.R. (1960) Anomalies of fertilization leading to triploidy. J. cell. comp. Physiol. 56, Suppl. 1, 1-15.

BEDFORD, J.M. (1967) Experimental requirement for capacitation and observations on ultrastructural changes in rabbit spermatozoa during fertilization. J. Reprod. Fert., Suppl. 2, 35-48.

BracketT, B.G. \& Williams, W. (1968) Fertilization of rabbit ova in a defined medium. Fert. Steril. 19, $144-155$.

BRADEN, A.W.H. (1953) Distribution of sperms in the genital tract of the female rabbit after coitus. Aust. J. biol. Sci. 6, 693-705.

Chang, M.C. (1959) Fertilization of rabbit ova in vitro. Nature, Lond. 184, 466-467.

Chang, M.C., Hanada, A. \& Hunt, D.M. (1971) Fertilization of denuded rabbit eggs in vitro by sperm recovered from the uterus and vagina. Nature, Lond. 232, 343-344.

DAuzier, L. \& ThibaulT, C. (1956) Recherche expérimentales sur la maturation des gametes males chez les mammifères, par l'étude de la fecondation in vitro de la lapine. Proc. $3 r d$ Int. Congr. Anim. Reprod. \& A.I., Cambridge, Section I, 58-61.

Fraser, L.R. \& Dandekar, P.V. (1973a) Fertilization of rabbit eggs in vitro without supplemental $\mathrm{CO}_{2}$ in the atmosphere. J. Reprod. Fert. 33, 159-161.

Fraser, L.R. \& Dandekar, P.V. (1973b) The effects of aging on in vitro fertilization of rabbit eggs and subsequent embryonic development. J. exp. Zool. 184, 303-312.

FRASER, L.R., DANDEKaR, P.V. \& VAIDYA, R.A. (1971) In vitro fertilization of tubal rabbit ova partially or totally denuded of follicular cells. Biol. Reprod. 4, 229-233.

Morton, D.B. \& Glover, T.D. (1974) Sperm transport in the female rabbit: the rôle of the cervix. J. Reprod. Fert. 38, 131-138.

Seitz, H.M., Rocha, G., Brackett, B.G. \& MastroiANNI, L. (1970) Influence of the oviduct on sperm capacitation in the rabbit. Fert. Steril. 21, 325328. 